\title{
Drosophila Trap1 protects against mitochondrial dysfunction in a PINK1/parkin model of Parkinson's disease
}

\author{
AC Costa ${ }^{1}$, SHY Loh ${ }^{*, 1}$ and L Miguel Martins ${ }^{\star, 1}$
}

Mitochondrial dysfunction caused by protein aggregation has been shown to have an important role in neurological diseases, such as Parkinson's disease (PD). Mitochondria have evolved at least two levels of defence mechanisms that ensure their integrity and the viability of their host cell. First, molecular quality control, through the upregulation of mitochondrial chaperones and proteases, guarantees the clearance of damaged proteins. Second, organellar quality control ensures the clearance of defective mitochondria through their selective autophagy. Studies in Drosophila have highlighted mitochondrial dysfunction linked with the loss of the PTEN-induced putative kinase 1 (PINK1) as a mechanism of PD pathogenesis. The mitochondrial chaperone TNF receptor-associated protein 1 (TRAP1) was recently reported to be a cellular substrate for the PINK1 kinase. Here, we characterise Drosophila Trap1 null mutants and describe the genetic analysis of Trap1 function with Pink1 and parkin. We show that loss of Trap1 results in a decrease in mitochondrial function and increased sensitivity to stress, and that its upregulation in neurons of Pink1 mutant rescues mitochondrial impairment. Additionally, the expression of Trap1 was able to partially rescue mitochondrial impairment in parkin mutant flies; and conversely, expression of parkin rescued mitochondrial impairment in Trap1 mutants. We conclude that Trap1 works downstream of Pink1 and in parallel with parkin in Drosophila, and that enhancing its function may ameliorate mitochondrial dysfunction and rescue neurodegeneration in PD.

Cell Death and Disease (2013) 4, e467; doi:10.1038/cddis.2012.205; published online 17 January 2013

Subject Category: Neuroscience

Accumulating evidence suggests that the disruption of mitochondrial function and dynamics contributes to age-related neurodegenerative diseases, such as Parkinson's disease (PD). ${ }^{1}$ Accordingly, cells have developed complex quality control mechanisms to cope with the different challenges constantly imposed on the integrity of mitochondria. ${ }^{2,3}$

Molecular quality control, the first level of the mitochondrial defence mechanism, involves the upregulation of nuclear genes that encode mitochondrial chaperones and proteases, ensuring the removal of misfolded and non-assembled polypeptides. This stress response is known as the mitochondrial unfolded-protein response $\left(\mathrm{UPR}^{\mathrm{mt}}\right){ }^{4}$

Furthermore, the highly dynamic nature of mitochondria facilitates quality control through fusion and fission. ${ }^{5,6}$ Thus, damaged mitochondria can fuse with healthy organelles to replenish stores of deficient components. When the damage is excessive, such processes are overwhelmed; mitochondria become depolarised, and their components are targeted for recycling through a specific form of autophagy, termed mitophagy. 7,8

Recent findings have demonstrated that PTEN-induced putative kinase 1 (PINK1) is capable of directly modulating the activity of chaperones. ${ }^{9,10}$ The TNF receptor-associated protein 1 (TRAP1) is a mitochondrial chaperone suggested to act as a cellular substrate of the PINK1 kinase. In cultured cells, PINK1 expression shows a protective effect against oxidative stress-induced apoptosis. Upon the induction of oxidative stress, TRAP1 is phosphorylated in a PINK1dependent manner, and this phosphorylation is necessary to confer resistance to apoptosis. ${ }^{10}$

TRAP1 is a member of the HSP90 family, which has been shown to protect cells from oxidative stress and apoptosis caused by different agents, ${ }^{11-14}$ and its upregulation in tumours has been associated with chemotherapy resistance. ${ }^{15,16}$

The fruit fly, Drosophila melanogaster, has recently emerged as a powerful model system for studying the links between mitochondrial dysfunction and parkinsonian neurodegeneration. ${ }^{17}$ In Drosophila, the loss of Pink1 leads to mitochondrial dysfunction associated with muscle degeneration and the loss of dopaminergic neurons. ${ }^{18,19}$ In addition, a decreased expression of Trap1 was found to enhance the loss of dopaminergic neurons in flies expressing a mutant form of human $\alpha$-synuclein that causes PD. ${ }^{20}$

Here, we describe the consequences of Trap1 lossof-function in Drosophila and its gain-of-function on mitochondrial integrity. We also genetically address the role of Trap 1 in mitochondrial quality control through the Pink1/parkin pathway.

\footnotetext{
${ }^{1}$ Cell Death Regulation Laboratory, MRC Toxicology Unit, Leicester, UK

*Corresponding author: LM Martins or SHY Loh, Cell Death Regulation Laboratory, MRC Toxicology Unit, Hodgkin Building, Lancaster Road, Leicester LE1 9HN, UK. Tel: + 441162525533 or + 44116223 1501; Fax: + 441162525616 or + 44116252 5616; E-mail: martins.Imiguel@gmail.com or shyl1@|e.ac.uk Keywords: Drosophila; mitochondria; Parkinson's disease; stress Abbreviations: ATP, adenosine triphosphate; PD, Parkinson's disease; ROS, reactive oxygen species Received 7.11.12; revised 5.12.12; accepted 6.12.12; Edited by G Melino.
} 


\section{Results}

Loss of Trap1 increases sensitivity to stress and causes a decline in mitochondrial function. To investigate the in vivo role of Trap1 in Drosophila, we first determined the consequences of its loss. We obtained Trap $1^{4}$ mutant flies, generated by an imprecise excision of the P-element insertion line $\mathrm{P}(\mathrm{EPgy} 2) \mathrm{Trap}^{\mathrm{EY} 10238}$. The excision deleted the majority of the Trap1 gene (Figure 1a). We next performed quantitative real-time RT-PCR and confirmed that Trap $1^{4}$ mutants show a total loss of Trap1 mRNA, and that its neighbouring genes were not affected by the imprecise excision of the P-element (Figure 1b). The Trap1 mutant flies were viable and developed to adulthood; however, they presented a significantly shorter lifespan compared with the controls (Figure 1c). Given the proposed role of TRAP1 as a mitochondrial chaperone, we decided to investigate the consequences of exposing Trap $1^{4}$ mutants to increased levels of stress. We observed a decreased viability of Trap $1^{4}$ mutants subjected to heat stress (Figure 1d). The Trap $1^{4}$ mutants were also more sensitive to paraquat, a pesticide linked to PD by epidemiological studies ${ }^{21}$ (Figure 1e), and to mitochondrial poisons such as rotenone (Figure 1f) and antimycin (Figure 1g). The Trap $1^{4}$ mutants showed an agedependent impaired climbing ability, suggesting a locomotor deficit (Figure 1h). In Pink1 mutant flies, locomotor defects result from mitochondrial impairment in the Drosophila skeletal muscles, including the indirect flight muscle. ${ }^{18,19}$
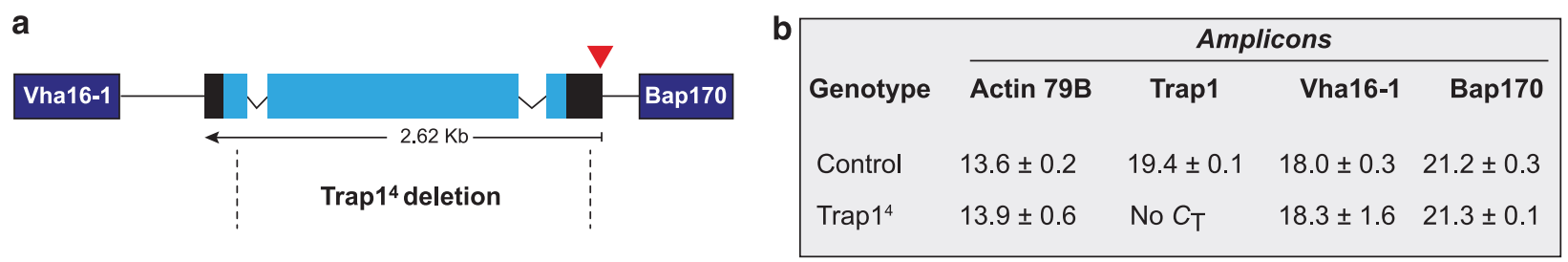

C

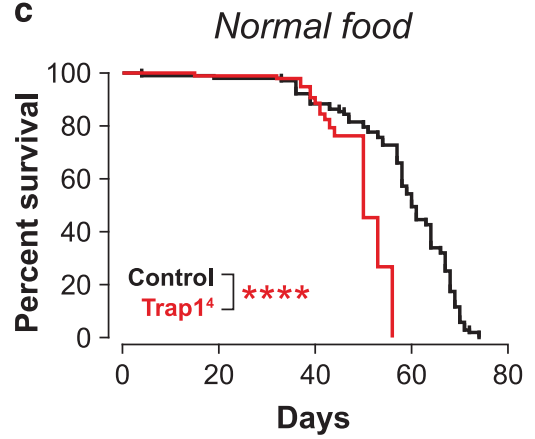

d

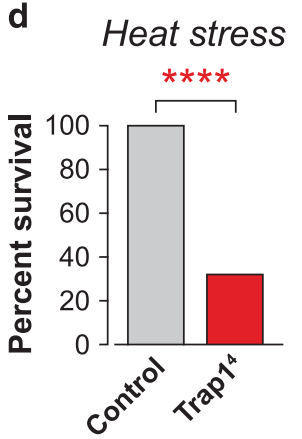

e

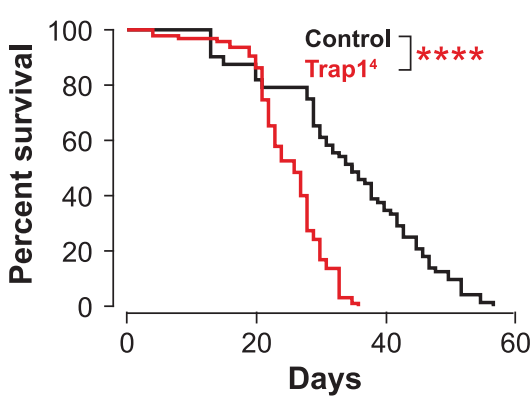

f

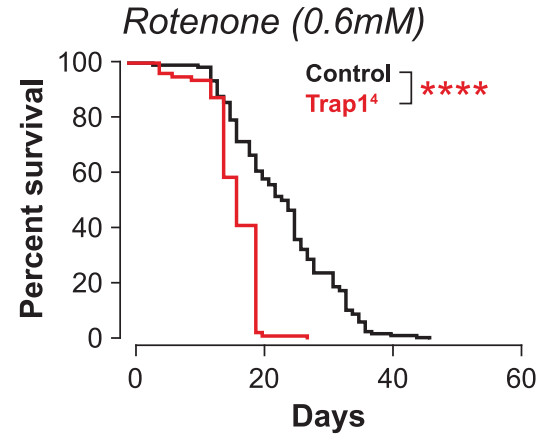

g

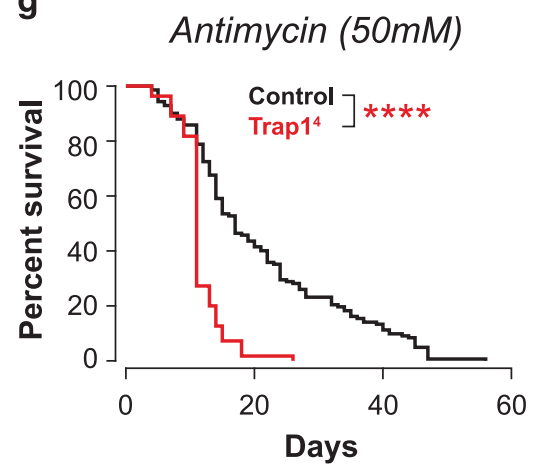

h

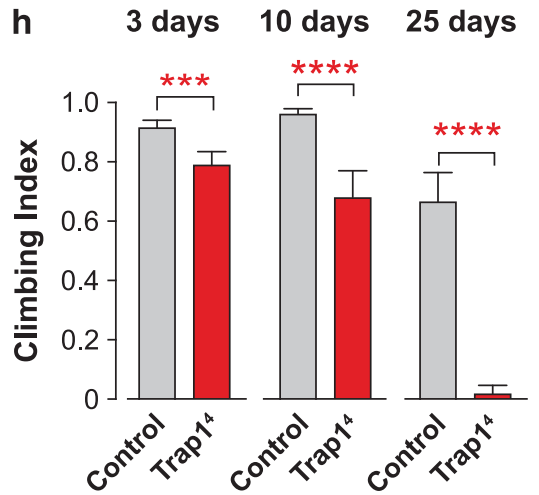

Figure 1 The loss of Trap1 in Drosophila causes motor impairment and an increased sensitivity to stress. (a) Genomic map of Trap1 (cytological location 42B2). Black, untranslated regions; light blue, exons. The P-element insertion (EY10238) is indicated by the red triangle. The neighbouring genes (Vha16-1 and Bap170) are indicated in dark blue. Trap $1^{4}$ deletion, delimited by the dashed lines, removes most of the Trap1 gene. (b) Analysis of the expression levels of Trap1 and its neighbouring genes. Expression levels were measured by real-time PCR in 3-day-old flies with the indicated genotypes (mean $\mathrm{Ct} \pm$ S.D., $n=4$ per genotype). Expression of actin was used as a control. No Ct for the Trap1 transcript was detected in Trap $1^{4}$ mutants. (c) Trap $1^{4}$ mutants (red) have a reduced lifespan compared with the controls (black). Fly viability was scored over a period of 75 days, using a minimum of 100 flies per genotype. The statistical significance is indicated (log-rank, Mantel-Cox test). (d-g) Trap1 mutant flies show enhanced sensitivity to stress. (d) Flies were subjected to heat stress, and viability was assessed after $24 \mathrm{~h}$. A total of 100 males (4 days old) were assayed for each genotype. The asterisks indicate significant values (Fisher's exact test, two-sided, alpha $<0.05)$. (e-g) Flies were maintained on food supplemented with the indicated drugs, and the viability was scored over a period of 60 days, using a minimum of 100 flies per genotype. The statistical significance is indicated by the asterisks (log-rank, Mantel-Cox test). (h) Trap $1^{4}$ mutants show a decrease in motor performance. Flies with the indicated genotypes and ages were tested using a standard climbing assay (mean \pm S.D., $n \geq 80$ flies for each genotype). The asterisks indicate significant values (two-way ANOVA with the Bonferroni multiple comparison test) 
a

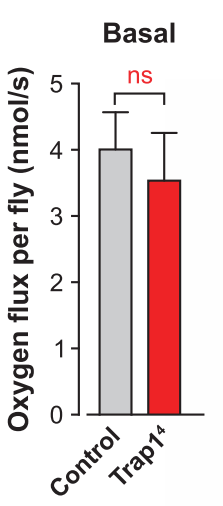

Coupled state 3

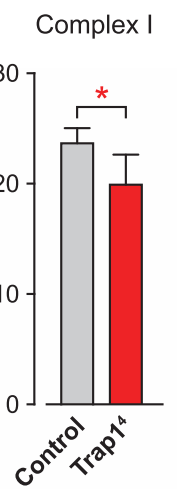

Complex II

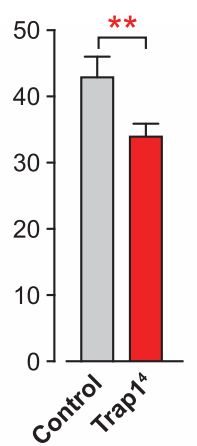

b

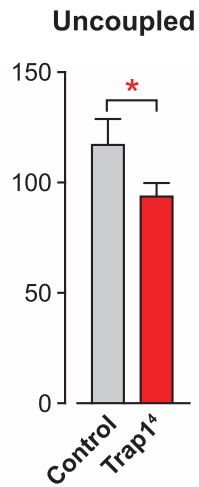

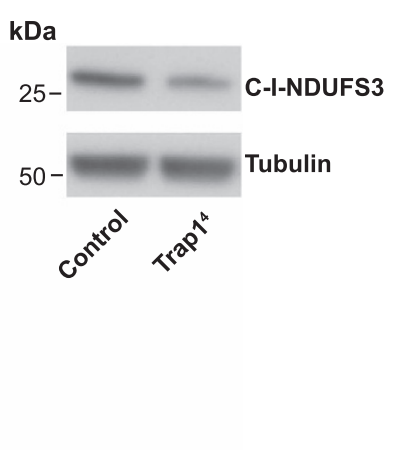

C

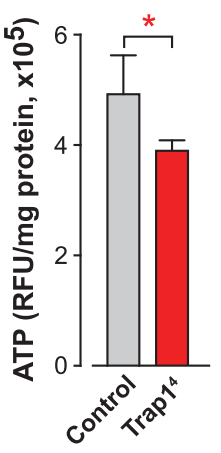

d
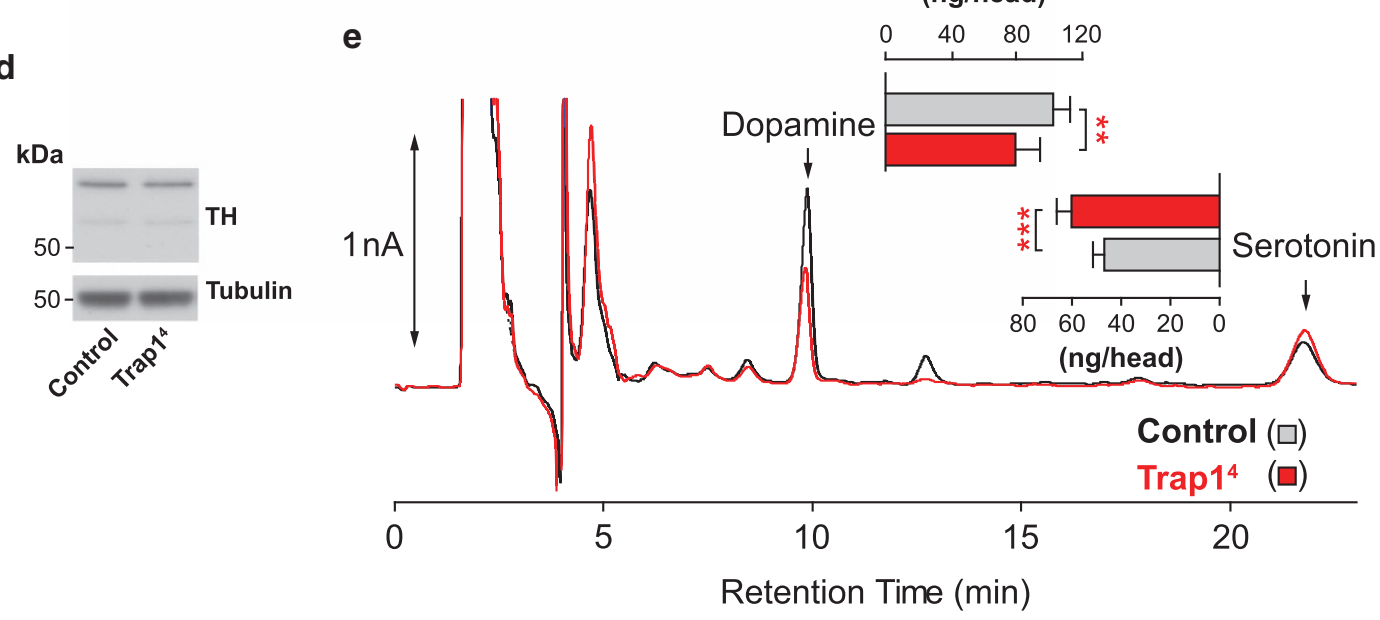

(ng/head)

Figure 2 The loss of Trap1 in Drosophila results in a reduction of mitochondrial function and brain dopamine levels. (a) Decreased respiration in Trap $1^{4}$ mutant flies. Activity was measured by high-resolution respirometry in 20-day-old flies. Data are shown as the means \pm S.D. ( $n \geq 4$ in each genotype). Significant values relative to the control are indicated by asterisks (two-tailed unpaired $t$ test). (b) Decreased complex I protein levels in Trap $1^{4}$ mutant flies. Whole-fly (20 days old) protein lysates were subjected to western blot analysis with the indicated antibodies. (c) Trap $1^{4}$ mutants have lower levels of ATP compared with the controls. The ATP levels were measured using a bioluminescent assay. Data are shown as the means \pm S.D. ( $n=4$ for each genotype). The statistical significance is indicated by asterisks (two-tailed unpaired $t$-test). (d) Analysis of TH levels in Trap1 mutant flies. Fly-head protein lysates from 20-day-old flies were used for western blotting analysis with the indicated antibodies. (e) Trap1 mutant flies have decreased dopamine and increased serotonin levels. Neurotransmitter levels were assessed by HPLC with electrochemical detection. Data are shown as the means \pm S.D. ( $n \geq 5$ for each genotype). The statistical significance is indicated by asterisks (two-tailed unpaired $t$-test)

To determine if the loss of Trap1 lead to mitochondrial impairment, we compared the respiration rates in the controls and mutants. This analysis revealed a significant decrease in the respiratory function of the Trap1 mutants (Figure 2a). Trap $1^{4}$ mutants also showed a decrease in the levels of the mitochondrial complex I (Figure 2b). Mitochondria are responsible for the production of the majority of cellular energy in the form of ATP. The measurement of ATP levels in Trap $1^{4}$ mutants revealed a significant decrease when compared with controls (Figure 2c). Taken together, these results suggest that the loss of the mitochondrial chaperone Trap1 results in a decrease in mitochondrial function, which is associated with a loss of ATP in adult flies. The loss of dopaminergic neurons can be indirectly assessed through the analysis of the expression levels of tyrosine hydroxylase (TH), an enzyme expressed in dopaminergic neurons. ${ }^{22}$ We failed to detect any differences in the $\mathrm{TH}$ levels of $\mathrm{Trap}^{4}$ mutants (Figure 2d); however measuring neurotransmitter levels in the heads of Trap $1^{4}$ mutants revealed a significant decrease in the dopamine content, compared to the controls (Figure 2e).

Trap1 expression enhances motor performance and protects against oxidative stress. Recently, TRAP1 was suggested to work downstream of PINK1 in the prevention of mitochondrial dysfunction associated with PD pathogenesis. ${ }^{10}$ We therefore decided to test the epistatic relationship between Trap1 and Pink1 in flies. We generated transgenic flies expressing full-length Trap1, and using UAS-GAL4 system ${ }^{23}$ we confirmed the expression of the transgene by western blot analysis (Figure 3a). To investigate its intracellular localisation, we crossed UAS-Trap1 flies with a transgenic line expressing mitochondria-targeted green-fluorescent protein (mitoGFP). This analysis revealed that Trap1 localises to the mitochondria (Figure 3b). To further investigate the phenotypic consequences of Trap1 expression, we compared the locomotor performance of Trap1 flies with that of controls using a climbing 


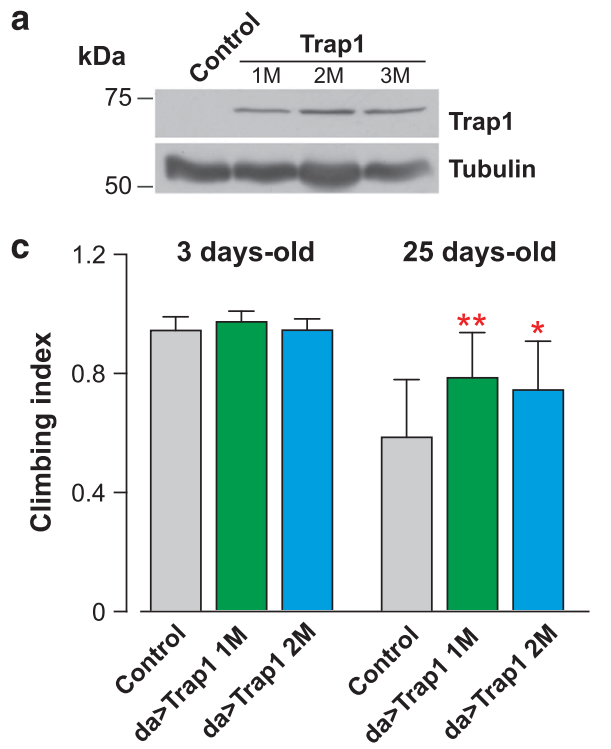

b

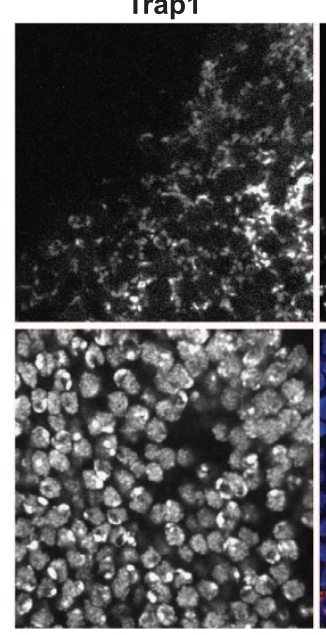

Nuclei

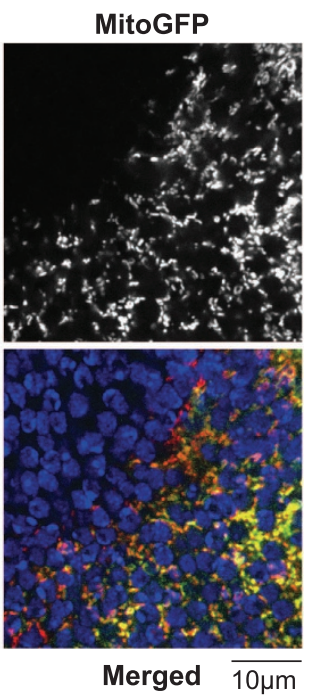

e

Normal food

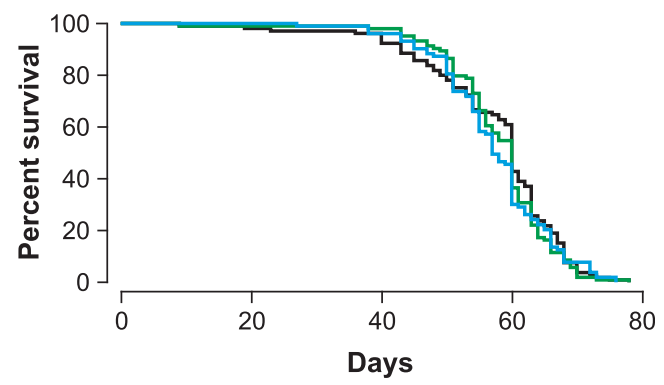

f

Paraquat (5mM)

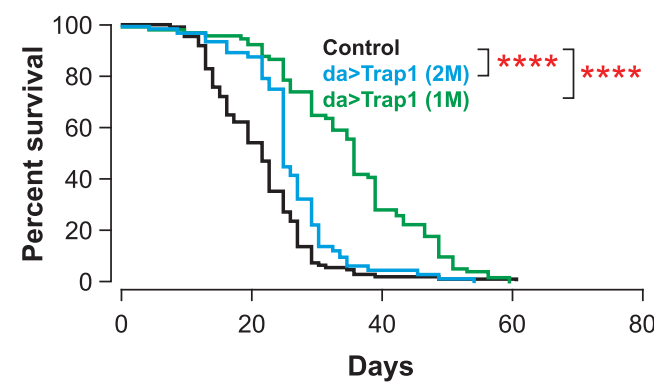

Figure 3 Expression of Drosophila Trap1 confers resistance to stress. (a) Analysis of the Trap1 expression levels in transgenic flies. Whole-fly lysates were analysed by western blotting with the indicated antibodies. (b) Trap1 localises to the mitochondria. Confocal analysis of the posterior compartment of larval wing discs coexpressing Trap1 and a mitoGFP under the control of the enGAL4 driver. In the merged colour image, red corresponds to Trap1, blue to nuclei and green to mitoGFP. (c) The expression of Trap1 results in increased motor performance. Flies with the indicated genotypes and ages were tested using a standard climbing assay (mean \pm S.D., $n \geq 120$ flies for each genotype). The asterisks indicate significant values relative to the control (two-way ANOVA with the Bonferroni multiple comparison test). (d) The expression of Trap1 reversed the decrease in motor performance in Trap $1^{4}$ mutants. Flies with the indicated genotypes and ages were tested using a standard climbing assay (mean \pm S.D., $n \geq 80$ flies for each genotype). The asterisks indicate significant values relative to the control (two-way ANOVA with the Bonferroni multiple comparison test). (e) Trap1 expression does not affect the total lifespan. A total of 100 flies per genotype were maintained on normal food for a period of 80 days. (f) Trap1-expressing flies (green and blue) show enhanced resistance to paraquat toxicity compared with the controls (black). Fly viability was scored over a period of 60 days using a minimum of 100 flies per genotype. The statistical significance is indicated by the asterisks (log-rank, Mantel-Cox test)

assay. This analysis revealed that Trap1 expression leads to a significantly enhanced climbing performance in aged flies (Figure 3c) and suppressed the climbing defects of Trap $1^{4}$ mutants (Figure $3 \mathrm{~d}$ ). We previously showed that treating flies with paraquat leads to the upregulation of mitochondrial chaperones as a means to protect mitochondria from the oxidative stress triggered by this pesticide. ${ }^{24}$ Additionally,
TRAP1 phosphorylation was reported to be enhanced in response to oxidative stress. ${ }^{10}$ Flies expressing Trap1 show a normal lifespan compared with the controls (Figure $3 e$ ). However, Trap1 expression significantly increased the lifespan of paraquat-treated flies (Figure $3 \mathrm{f}$ ), indicating that the expression of this mitochondrial chaperone protects against oxidative stress in vivo. 

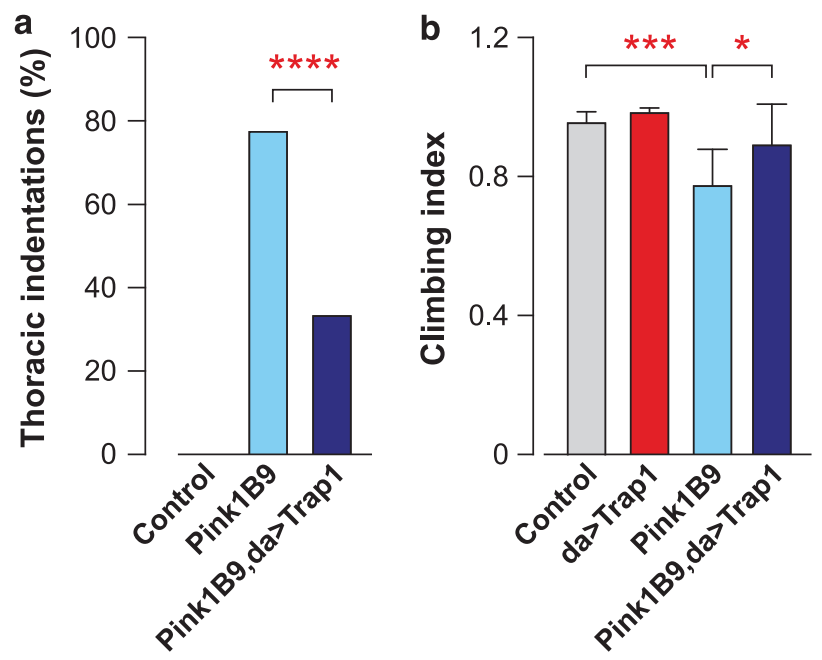

C

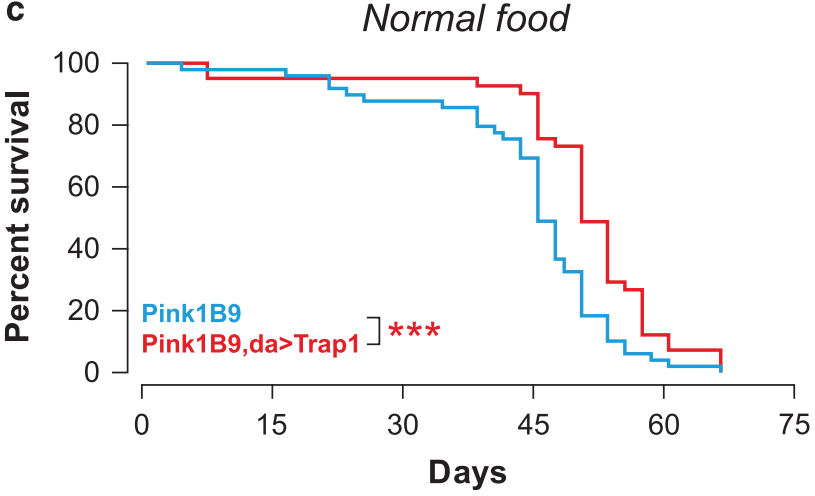

d Paraquat $(5 \mathrm{mM})$

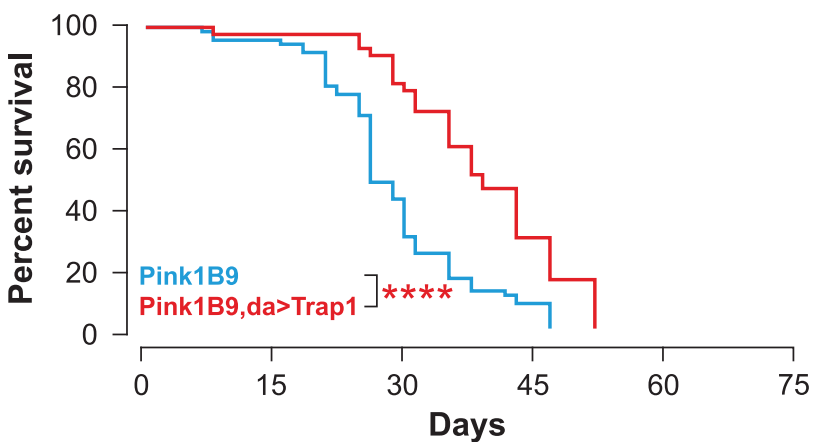

Figure 4 Trap1 gain-of-function rescues Pink1 mutant flies. (a) Expression of Trap1 rescues the thoracic defects of Pink $1^{B 9}$ mutants. Thoracic indentations were counted up to $24 \mathrm{~h}$ after eclosion ( $n>900$ for each genotype). The asterisks indicate significance $\left(\chi^{2}\right.$, two-sided, alpha $\left.<0.05\right)$. (b) Trap1 expression suppresses motor impairment in the Pink $1^{B 9}$ mutants. 10-day-old flies with the indicated genotypes were tested using a standard climbing assay (mean \pm S.D., $n=120$ flies per genotype). The statistical significance relative to $P$ ink $1^{B 9}$ is indicated by asterisks (one-way ANOVA with Dunnett's multiple comparison test). (c and d) Trap1 expression enhances the lifespan of Pink $1^{B 9}$ mutants. A minimum of 80 flies per genotype were maintained on either normal (c) or paraquat-containing food (d). Fly viability was scored over a period of 70 days. The statistical significance is indicated by the asterisks (log-rank, Mantel-Cox test)

Expression of Trap1 suppresses mitochondrial dysfunction in Pink1 mutants. The expression of TRAP1 in cultured cells was shown to be required for PINK1-depen- dent inhibition of oxidative stress-induced cytochrome $c$ release and cell death. ${ }^{10}$ The mitochondrial dysfunction in Pink1 mutants is associated with muscle pathology, affecting the indirect flight muscles, leading to the appearance of thoracic indentations and impaired motor performance. ${ }^{18,19}$ The expression of Trap1 partially rescued the degree of thoracic indentations (Figure 4a) and the impaired motor performance in the Pink $1^{B 9}$ mutants (Figure 4b). Mitochondrial defects present in the Pink1 mutants are also associated with a decreased lifespan. ${ }^{18}$ We noted that the expression of Trap1 increased the lifespan of the Pink1 mutants (Figure 4c) and decreased their sensitivity to paraquat (Figure 4d). Taken together, these observations suggest that the ubiquitous expression of Trap1 protects against the phenotypic consequences of Pink1 mutations, and that Trap1 acts genetically downstream of Pink1. The mitochondrial dysfunction in the Pink1 mutant flies is reflected by a loss of mitochondrial proteins and decreased respiration. By expressing Trap1 in Pink1 ${ }^{B 9}$ mutants, we observed a recovery of the mitochondrial protein content, indirectly assessed through the analysis of the levels of a complex I subunit (Figure 5a); a partial recovery of the respiration rates (Figure $5 b$ ) and ATP levels (Figure $5 c$ ). Taken together, these observations indicate that Trap1 expression results in a recovery of the mitochondrial function in Pink1 mutants.

Neuronal expression of Trap1 is sufficient to revert the phenotype of Pink1 mutants. The genetic removal of the function of Drosophila Pink1 results in apoptotic muscle degeneration and a decrease in the brain dopamine levels as a consequence of the degeneration of dopaminergic neurons. ${ }^{19}$

The loss of mitochondria at neuronal synapses causes defects in neurotransmission of the Drosophila neuromuscular junction (NMJ). ${ }^{25}$ Given that one of the most important features of Pink1 mutant flies is the degeneration of both the indirect flight muscle and dopaminergic neurons, we next tested whether the degeneration of the indirect flight muscle involves a presynaptic component, and is therefore preceded by neuronal loss.

We detected a decrease in the TH levels in Pink $1^{B 9}$ mutants that was reversed upon the expression of Trap1 using a panneural elav-GAL4 driver (Figure 6a). This result indicates that the neuronal expression of Trap1 can suppress neurodegeneration in Pink1 mutant flies. Next, we evaluated the consequence of the neuronal expression of Trap1 on phenotypes associated with muscle degeneration. The neuronal expression of Trap1 was sufficient to suppress muscle degeneration in Pink $1^{B 9}$ mutants, which was reflected in a decrease in the degree of thoracic indentations (Figure $6 b$ ) and an improved climbing performance (Figure 6c). Finally, the neuronal expression of Trap1 significantly reversed the respiration deficit present in Pink1 mutant flies (Figure 6d). Taken together, these results indicate that the neuronal rescue of mitochondrial function in Pink1 mutants by Trap1 is sufficient to suppress muscle degeneration and motor impairment.

Genetic epistasis analysis of parkin and Trap1. Upon mitochondrial damage, PINK1 has been shown to recruit 


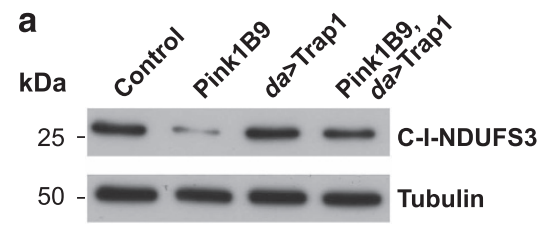

C

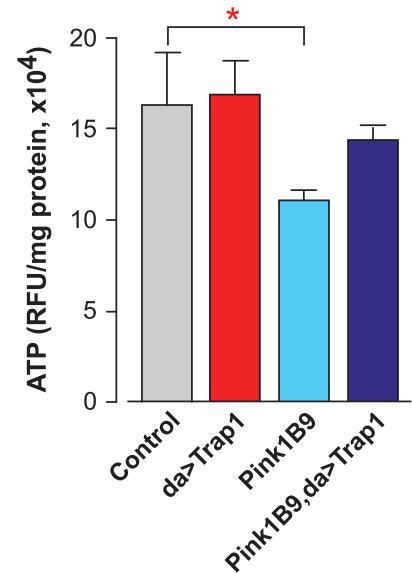

b

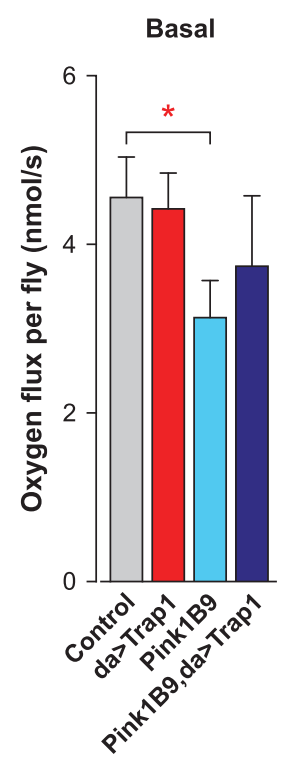

Coupled state 3

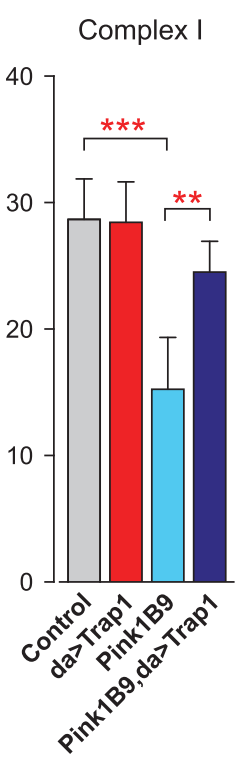

\section{Complex II}

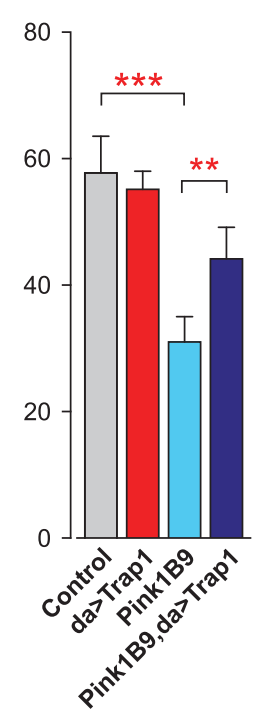

Uncoupled

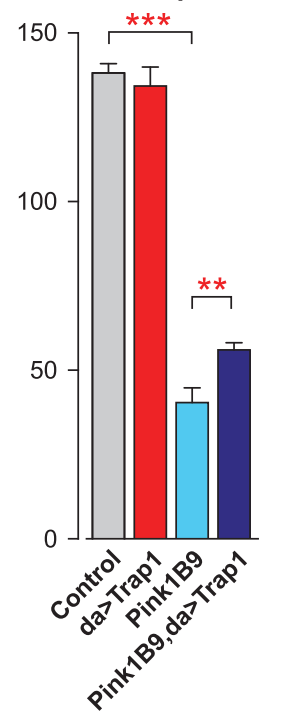

Figure 5 Drosophila Trap1 reverses mitochondrial dysfunction in Pink1 mutants. (a) Trap1 expression restores the mitochondrial complex I protein levels in the Pink $1^{B 9}$ mutant flies. Whole-fly protein lysates were used for western blotting analysis with the indicated antibodies. (b) Trap1 expression partially rescues the mitochondrial respiratory defects in the Pink $1^{B 9}$ mutants. Measurements of state three respiration of complex I and complex II, and complex II uncoupled respiration by high-resolution respirometry. The data are shown as the means \pm S.D. ( $n=6$ for each genotype). The statistical significance is indicated by the asterisks (one-way ANOVA with Dunnett's multiple comparison test). (c) Expression of Trap1 increased the ATP levels in the Pink $1^{B 9}$ mutants. ATP levels were measured using a bioluminescent assay. Data are shown as the means \pm S.D. ( $n=4$ for each genotype). The statistical significance is indicated by the asterisks (one-way ANOVA with Dunnett's multiple comparison test relative to the control)

a

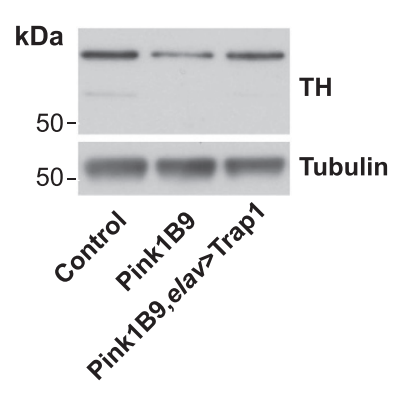

b

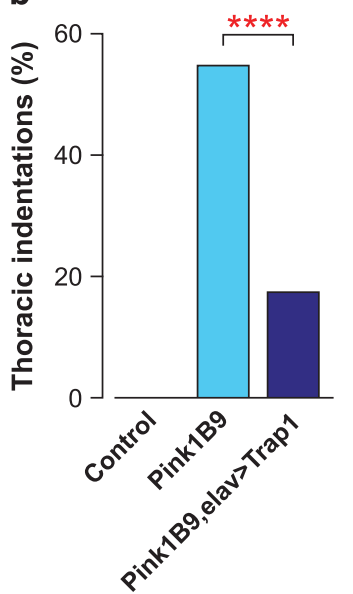

C

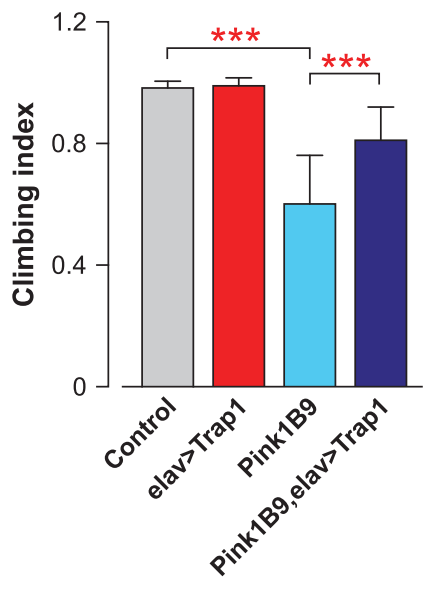

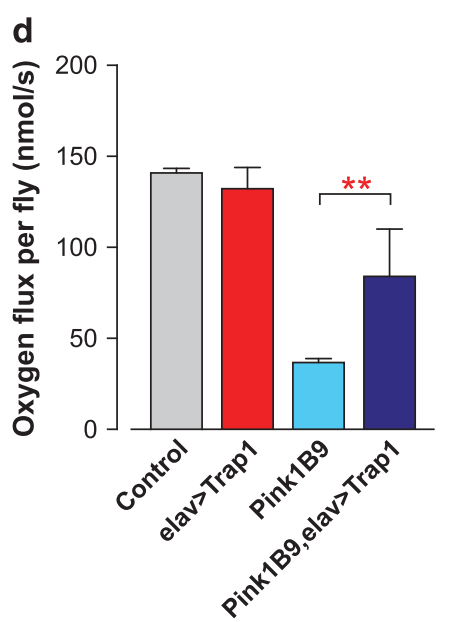

Figure 6 Targeted neuronal expression of Trap1 rescues mitochondrial dysfunction in Pink1 mutants. (a) Decreased TH levels observed in the Pink $1^{B 9}$ mutants can be partially restored by the expression of Trap1 in neurons. Fly-head protein lysates from 20-day-old flies were used for western blotting analysis with the indicated antibodies. (b) Neuronal expression of Trap 1 is capable of diminishing the degree of thoracic indentations in the Pink $1^{B 9}$ mutants. Thoracic indentations were counted up to $24 \mathrm{~h}$ after eclosion $\left(n>300\right.$ for each genotype). The asterisks indicate the significance relative to $P$ ink $1^{B 9}\left(\chi^{2}\right.$, two-sided, alpha $\left.<0.05\right)$. (c) Targeted neuronal expression of Trap1 improves the climbing performance of $P$ ink $1^{B 9}$ mutants. 10-day-old flies with the indicated genotypes were tested using a standard climbing assay (mean \pm S.D., $n=120$ flies per genotype). The statistical significance relative to Pink $1^{B 9}$ is indicated by the asterisks (one-way ANOVA with Dunnett's multiple comparison test). (d) Expression of Trap1 specifically in neurons is sufficient to improve mitochondrial respiration in the Pink $1^{B 9}$ mutants. Complex II-uncoupled respiration was measured by high-resolution respirometry. Data are shown as the means \pm S.D. ( $n=6$ for each genotype). The statistical significance is indicated by the asterisks (one-way ANOVA with Dunnett's multiple comparison test relative to the Pink $1^{B 9}$ mutant)

Parkin, a cytosolic E3 ubiquitin ligase, to damaged mitochondria. ${ }^{26,27}$ Parkin recruitment to damaged mitochondria has been proposed to cause the ubiquitination of the profusion factor Mfn to label terminally damaged mitochondria for autophagic degradation, ${ }^{28}$ a process that has been defined as organellar quality control. ${ }^{3,29}$ The importance of Parkin as 
a downstream effector of PINK1 was confirmed in a series of genetic studies in Drosophila, which showed that the expression of parkin could rescue the phenotype of Pink1 mutant flies. ${ }^{18,19}$ To address the epistatic relationship between parkin and Trap1, we first tested whether Trap1 expression could also rescue the parkin mutant phenotype. The expression of Trap1 partially rescued the degree of thoracic indentations (Figure 7a) but not the impaired motor performance in the parkin mutants (Figure 7b). We also observed that the expression of Trap1 had a modest effect on the suppression of the ATP loss present in parkin mutants (Figure 7c) and caused a recovery in the mitochondrial protein content, indirectly assessed through the analysis of the levels of a complex I subunit (Figure 7d). Moreover, Trap1 expression in parkin mutants led to an increased level of survival in flies maintained on normal food (Figure 7e) and on paraquat-containing food (Figure 7f). We next tested whether parkin expression could also rescue the Trap1 mutant phenotype. When we expressed parkin in Trap $1^{4}$ mutant flies, we observed a significant suppression of their climbing defects (Figure $7 \mathrm{~g}$ ), as well as a marked recovery of the levels of mitochondrial complex I (Figure 7h). Taken together, these results show that the enhanced expression of Trap1 can partially compensate for the loss of parkin, and reciprocally, the enhanced expression of parkin can also ameliorate some of the Trap1 mutant-associated phenotypes. This suggests that both Trap1 and parkin act in a parallel pathway that modulates mitochondrial competence.

\section{Discussion}

We have characterised the Drosophila orthologue of the TRAP1 chaperone and found that, similar to its mammalian counterpart, it localises to the mitochondria. Using a loss-offunction approach, through the generation of Trap1 mutant flies that lack Trap1 expression, we determined that its loss compromises mitochondrial function. Mutations in either Pink1 or parkin influence mitochondrial dynamics and function, causing an impairment of fission, which affects male fertility by compromising the normal development of mitochondrial derivatives (also known as nebenkern). ${ }^{18,19,30,31}$ During spermatogenesis, Drosophila mitochondria undergo significant morphological changes that require fusion to form a large spherical nebenkern and subsequent fission when the nebenkern unfurls to yield two mitochondrial derivatives. ${ }^{32}$ The Trap 1 mutants developed normally to adulthood and were both viable and fertile, suggesting that their mitochondrial defects do not compromise the mitochondrial fission and fusion processes required for normal spermatogenesis.

Although Trap1 mutants show no defects in development, they had a decreased lifespan, progressive locomotor defects and a decrease in the brain dopamine levels. Taken together, these results suggest that this mitochondrial chaperone is important to minimise the age-dependent decline of mitochondrial function in post-mitotic cells, such as muscle cells and neurons, which rely heavily on mitochondrial function. The milder phenotype of Trap1 mutants compared with the Pink1 mutants suggest that other effectors of molecular quality control acting downstream of Pink 1 can compensate for the potential defects caused by the loss of Trap1. In this respect, the serine protease $\mathrm{HtrA} 2$, a downstream target of PINK $1^{9,33}$ involved in mitochondrial molecular quality control, ${ }^{34}$ could potentially partially compensate for any disturbances in protein folding in the intermembrane space of mitochondria.

The expression of Trap1 was sufficient to enhance the climbing performance of aged flies and promote resistance to oxidative stress (Figure 3). Mitochondria in skeletal muscles are highly specialised towards energy production for contractile activity, ${ }^{35}$ and therefore, the expression of this chaperone might contribute to the maintenance of mitochondrial performance in tissues with high-energy demand, such as skeletal muscles, thereby enhancing the motor performance.

Our results indicate that the neuronal expression of Trap1 in Pink1 mutants is sufficient to suppress muscle degeneration. Mitochondria have been shown to be concentrated in both the presynaptic nerve terminal and the postsynaptic endplates in muscle, ${ }^{36}$ indicating that a decline in mitochondrial bioenergetic function may affect synaptic transmission and result in a loss of NMJ innervation. Our data suggest that mitochondrial dysfunction at the presynaptic terminal of the $\mathrm{NMJ}$ is a major determinant of the pathologies of the skeletal muscle present in the Pink1 mutants, and that the compromise of the motor neuron function in the Pink1 mutants causes muscle dysfunction; therefore, is likely to precede muscle degeneration.

In Drosophila, the expression of parkin can rescue the phenotype of Pink1 mutants, presumably by enhancing the organellar quality control through autophagic disposal of

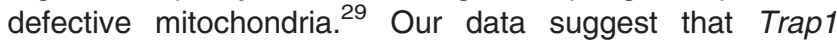
expression can partially compensate for the mitochondrial defects present in parkin mutant flies. Given that Trap1 is likely to act as a molecular chaperone in the clearance of misfolded proteins, its expression may lead to enhanced molecular quality control in mitochondria, and therefore decrease the demand for organellar quality control as a clearance mechanism for mitochondria overwhelmed by excessive protein misfolding. Enhanced expression of Trap1 may in turn decrease the demand on organellar quality control in parkin mutants, leading to a decrease in the degree of dysfunctional mitochondria in these insects. We also observed that the expression of parkin was capable of suppressing some of the phenotypic consequences associated with Trap1 mutations. This suggests that both the molecular and organellar pathways for mitochondrial quality control work in parallel, and the enhancement of either one of these can relieve pressure on the other and contribute to a better mitochondrial health.

\section{Materials and Methods}

Antibodies. The following primary antibodies were used in this study: antiTRAP1 mouse IgG (clone 30) (612342), BD Transduction Laboratories, Franklin Lakes, NJ, USA; anti-NDUFS3 mouse monoclonal (ab14711), Mitosciences, Eugene, OR, USA; anti-TH rabbit polyclonal (AB152), Millipore (Billerica, MA, USA); and anti- $\alpha$-tubulin IgG1 clone B-5-1-2, Sigma-Aldrich (St Louis, MO, USA).

Genetics and Drosophila strains. Transgenic strains with UAS-Trap1 were generated by P-element-mediated transformation. Transgenic flies were generated at BestGene Inc. (Chino Hills, CA, USA). Three independent transgenic lines were obtained, and all were tested for protein expression. Pink $1^{B 9}$, park ${ }^{25}$, UAS parkin ${ }^{62 B}$ and da-GAL4 flies were a gift from A Withworth (MRC, Centre for 
a

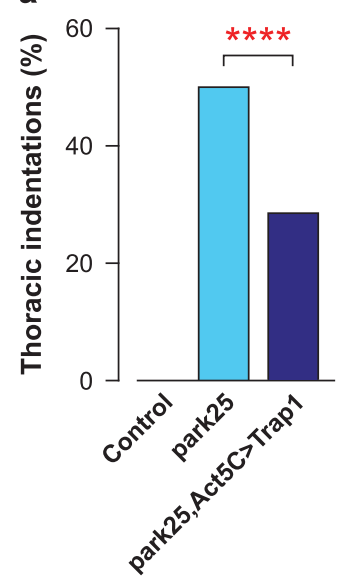

b

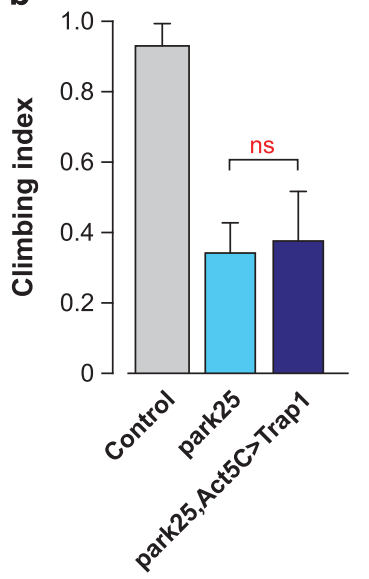

C

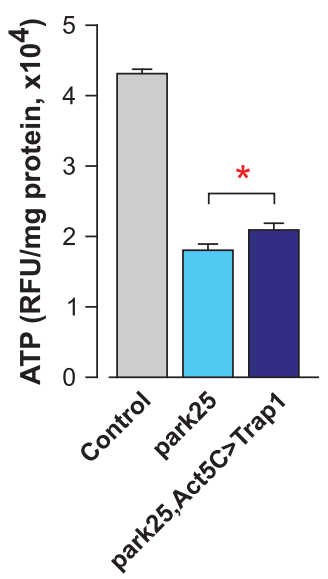

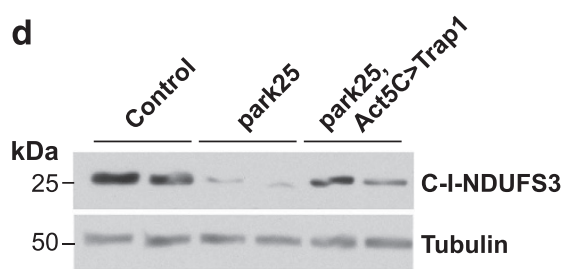

e

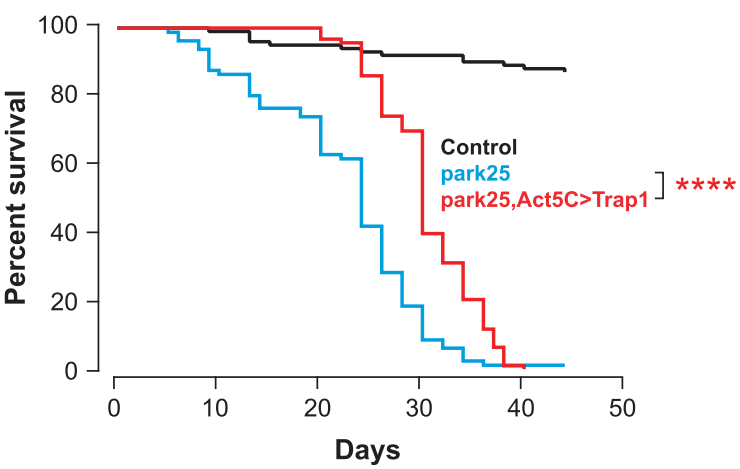

f

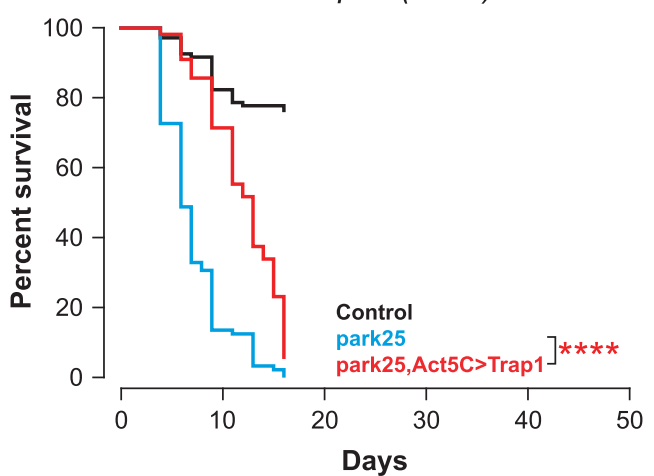

9 3 days-old

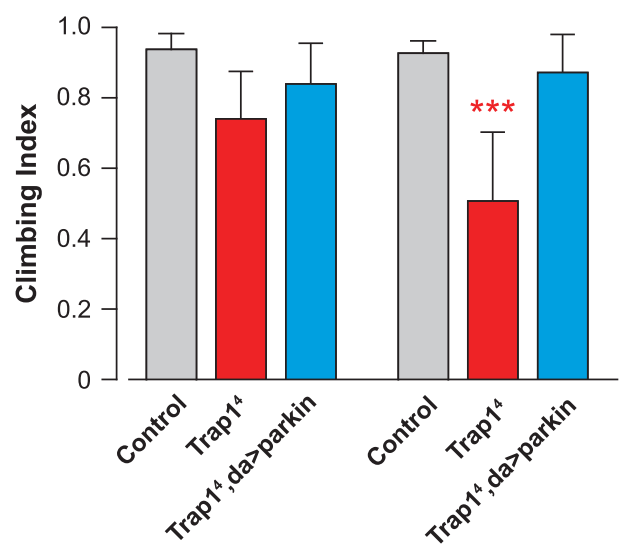

h kDa

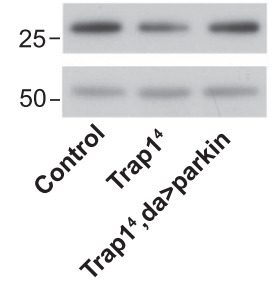

C-I-NDUFS3

Tubulin

Figure 7 Epistatic relationship between Trap1 and parkin. (a) Trap1 expression decreases the degree of thoracic indentations in parkin mutant flies, park ${ }^{25}$. Thoracic indentations were counted up to $24 \mathrm{~h}$ after eclosion $\left(n>250\right.$ for each genotype). The asterisks indicate the significance $\left(\chi^{2}\right.$, two-sided, alpha $\left.<0.05\right)$. (b) The expression of Trap1 fails to rescue the climbing defect of the park ${ }^{25}$ mutants. 3-day-old flies with the indicated genotypes were tested using a standard climbing assay (mean \pm S.D., $n>120$ flies per genotype). (c) Trap1 expression increases the levels of ATP in the park ${ }^{25}$ mutants. The ATP levels were measured using a bioluminescent assay. Data are shown as the means \pm S.D. ( $n=4$ for each genotype). The statistical significance is indicated by the asterisks (one-way ANOVA with Dunnett's multiple comparison test relative to park ${ }^{25}$ ). (d) Trap1 expression restores the mitochondrial complex I protein levels in the park ${ }^{25}$ mutant flies. Whole-fly protein lysates were used for western blotting analysis with the indicated antibodies. (e) Trap1 expression enhances the lifespan of the park ${ }^{25}$ mutants. Fly viability was scored over a period of 40 days using a minimum of 100 flies per genotype. The statistical significance is indicated by the asterisks (log-rank, Mantel-Cox test). (f) Expression of Trap1 increases the resistance of park $k^{25}$ mutants to paraquat-induced stress. Fly viability was scored over a period of 15 days using a minimum of 80 flies per genotype. The statistical significance is indicated by the asterisks (log-rank, Mantel-Cox test). (g) The expression of parkin rescues the climbing defects of Trap1 ${ }^{4}$ mutants. Flies with the indicated genotypes and ages were tested using a standard climbing assay (mean \pm S.D., $n>60$ flies per genotype). The asterisks indicate significant values (two-way ANOVA with the Bonferroni multiple comparison test). (h) parkin expression restores the mitochondrial complex I protein levels in Trap $1^{4}$ mutant flies. Whole-fly protein lysates from 20-day-old flies were used for western blotting analysis with the indicated antibodies 
Developmental and Biomedical Genetics, University of Sheffield, Sheffield, UK). Trap $1^{4}$ line was kindly provided by Dr. JE Treisman (New York University School of Medicine, NY, USA). w1118, Act5C-GAL4, enGAL4 and elav-GAL4 lines were obtained from Bloomington Stock Centre (Indiana University, Bloomington, IN, USA). Fly stocks and crosses were maintained on standard cornmeal agar media at $25^{\circ} \mathrm{C}$. The genotypes of the flies used in each individual figure are listed in Supplementary Table S1.

Molecular biology. Expression constructs encoding Drosophila Trap1 sequence were generated by polymerase chain reaction using appropriate adaptor primers. The PCR fragments were cloned into the GAL4-responsive pUAST expression vector for the generation of transgenic fly strains.

HPLC measurement of dopamine and serotonin. For sample preparation, 15 male fly heads (20 days old) were dissected under mild $\mathrm{CO}_{2}$ anaesthesia, and quickly frozen in dry ice. The heads were then homogenised in $50 \mu \mathrm{l}$ chilled $0.1 \mathrm{M}$ perchloric acid using a motorised, hand-held tissue homogeniser. The chilled homogenates of fly heads were filtered through a lowbinding Durapore $(0.22 \mu \mathrm{m})$ PVDF membrane using Ultrafree-MC centrifugal devices. The dopamine and serotonin measurements were performed immediately after sample preparation. Supernatant fluid $(30 \mu \mathrm{l})$ was eluted at a flow rate of $50 \mu \mathrm{l} / \mathrm{min}$ through a $150 \times 1.0 \mathrm{~mm} \mathrm{C} 18$ column. The mobile phase contained $50 \mathrm{mM}$ phosphoric acid, $8 \mathrm{mM} \mathrm{NaCl}, 0.1 \mathrm{mM}$ EDTA, $12.5 \%$ (v/v) methanol, $500 \mathrm{mg} / \mathrm{l}$ octanesulfonic acid (OSA) (pH 6.0). Analysis was performed using an Alexys LC-EC system equipped with a DECADE II electrochemical detector.

Immunofluorescence and confocal microscopy. Third instar larvae were dissected, fixed in $4 \%$ paraformaldehyde, stained with the anti-TRAP1 antibody and incubated with Hoechst 33342. Wing imaginal discs were mounted on glass slides with Vectashield (Vector Laboratories, Burlingame, CA, USA) and imaged on a Zeiss LSM510 confocal microscope.

Statistical analysis. The data are presented as mean values, and the error bars indicate \pm S.D. Inferential statistical analysis was performed using the Prism and StatMate software packages (www.graphpad.com). The significance level is indicated as ${ }^{* * * *}$ for $P<0.0001,{ }^{* * *}$ for $P<0.001,{ }^{* *}$ for $P<0.01,{ }^{*}$ for $P<0.05$ and ns for $P>0.05$

ATP assays. Five male flies ( 10 or 20 days old) were homogenised in $100 \mu \mathrm{l}$ of $6 \mathrm{M}$ guanidine- $\mathrm{HCl}$ in extraction buffer ( $100 \mathrm{mM}$ Tris and $4 \mathrm{mM}$ EDTA, pH 7.8 ) to inhibit ATPases.

Homogenised samples were subjected to rapid freezing in liquid nitrogen, followed by boiling for $5 \mathrm{~min}$. Samples were cleared by centrifugation, and supernatant was diluted $(1 / 50)$ with extraction buffer and mixed with a luminescent solution (CellTiter-Glo Luminescent Cell Viability Assay, Promega, Fitchburg, WI, USA). Luminescence was measured on an Infinite M200Pro multifunction reader (TECAN, Männedorf, Switzerland). The relative ATP levels were calculated by dividing the luminescence by the total protein concentration, which was determined by the Bradford method.

Climbing assays. The climbing assays were performed as previously described, ${ }^{37}$ using a countercurrent apparatus developed initially for phototaxis experiments. A total of 20 male flies were placed into the first chamber, taped to the bottom and then given $20 \mathrm{~s}$ to climb a distance of $10 \mathrm{~cm}$. Those flies that successfully climbed $10 \mathrm{~cm}$ or beyond in $20 \mathrm{~s}$ were then shifted to a new chamber, and both sets of flies were given another opportunity to climb the 10-cm distance. This procedure was repeated a total of five times. After five trials, the number of flies in each chamber was counted. At least 120 flies were used for each genotype tested.

Respirometry analysis. Mitochondrial respiration was assayed at $37^{\circ} \mathrm{C}$ by high-resolution respirometry using an OROBOROS Oxygraph. DatLab software package (OROBOROS, Innsbruck, Austria) was used for the data acquisition (2-s-time intervals) and analysis, including the calculation of the time derivative of the oxygen concentration, signal deconvolution dependent on the response time of the oxygen sensor and correction for instrumental background oxygen flux. Respiration was assayed by homogenising two flies using a pestle in MiR05

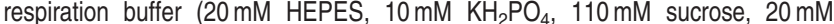
taurine, $60 \mathrm{mM} \mathrm{K}$-lactobionate, $0.5 \mathrm{mM} \mathrm{EGTA}, 3 \mathrm{mM} \mathrm{MgCl}$, $1 \mathrm{~g} / \mathrm{l}$ fatty acid-free $\mathrm{BSA}$ ). The basal respiration rates were assayed in the absence of exogenous respiration substrates. For coupled (state 3 ) assays, complex I activity was assayed in MiR05 respiration buffer in the presence of $2 \mathrm{mM}$ malate, $10 \mathrm{mM}$ glutamate and $5 \mathrm{mM}$ ADP. Complex $\|$ was assayed in respiration buffer supplemented with $1 \mathrm{mM}$ rotenone, $10 \mathrm{mM}$ succinate and $5 \mathrm{mM}$ ADP. ADP was not added for the measurement of respiration in uncoupled mitochondria obtained by one freeze (liquid nitrogen)-thaw cycle of the fly tissue.

Lifespan analysis. Groups of 10 newly eclosed males of each genotype were placed into separate vials with food and maintained at $25^{\circ} \mathrm{C}$. The flies were transferred to vials containing fresh food every 2-3 days, and the number of dead flies was recorded. The data are presented as Kaplan-Meier survival distributions, and the significance was determined by log-rank tests.

Drug treatments. All the drugs used in this study were incorporated into the fly food. The concentrations of each of the drugs in the food were the following: $5 \mathrm{mM}$ for paraquat, $600 \mu \mathrm{M}$ for rotenone and $50 \mathrm{mM}$ for antimycin A. The newly eclosed males were placed with normal food in groups of 10 for 1 day prior to their transfer to vials containing drug. The flies were transferred to new vials every 2-3 days, and the number of dead flies was recorded. The assay was completed after all the flies died. A minimum of 80 flies per genotype were analysed. The results are presented as Kaplan-Meier survival distributions, and the significance was determined by log-rank tests.

Preparation of Drosophila protein lysates and western blot analysis. Single adult flies (3,10 or 20 days old) were homogenised using a motorised pestle in lysis buffer $(0.1 \%$ Triton-X100, $10 \mathrm{mM}$ EDTA, $1 \mathrm{mM}$ DTT, $100 \mathrm{mM} \mathrm{KCl}, 20 \mathrm{mM}$ HEPES (pH 7.5), $5 \%$ glycerol, $1 \mu \mathrm{g} / \mathrm{ml}$ chymostatin, $1 \mu \mathrm{g} / \mathrm{ml}$ leupeptin, $1 \mu \mathrm{g} / \mathrm{ml}$ pepstatin $\mathrm{A}$ and $1 \mu \mathrm{g} / \mathrm{ml}$ antipain). The lysates were subsequently cleared by centrifugation. The proteins were separated on 12 or $10 \%$ SDS-PAGE gels and transferred to Immobilon PVDF membranes (Millipore). The membranes were blocked in $5 \%$ milk and then incubated with the indicated primary antibody prior to incubation with the appropriate HRP-conjugated secondary antibody. The antibody complexes were visualised by enhanced chemiluminescence $(E C L)$. The results are representative of at least three biological replicates per genotype.

RNA extraction and quantitative real-time RT-PCR. The isolation of total RNA from three adult flies per sample was performed using the RNeasy Mini Kit (Qiagen, Hilden, Germany). Quantitative real-time RT-PCR was performed on an $\mathrm{M} \times 4000$ (Stratagene, La Jolla, CA, USA) real-time cycler using the QuantiTect SYBR Green RT-PCR system (Qiagen). Primers used (QuantiTect Primer Assay, Qiagen) were Cat. No. QT00967393 (actin 79B, used as a housekeeping gene), Cat. No. QT00940520 (Trap1), Cat. No. QT00501130, (Vha16-1) and Cat. No. QT00940527, (Bap170). The quantification was performed using the comparative $\mathrm{Ct}$ method. ${ }^{38}$

Heat stress. For heat sensitivity, 4-day-old male flies were divided into groups of 15 and placed in empty vials (no food or water). The flies were maintained at $25^{\circ} \mathrm{C}$ for $30 \mathrm{~min}$, after which the thermo stress was applied by placing the vials in a water bath at $37^{\circ} \mathrm{C}$ for $1 \mathrm{~h}$. This step was followed by a 30 -min recovery at $25^{\circ} \mathrm{C}$ and a new heat stress for $1 \mathrm{~h}$ at $37^{\circ} \mathrm{C}$. The flies were then transferred to vials containing fresh food and maintained at $25^{\circ} \mathrm{C}$. The number of living flies was recorded $24 \mathrm{~h}$ later. A total of 100 flies per genotype were tested for thermo stress.

\section{Conflict of Interest}

The authors declare no conflict of interest.

Acknowledgements. We would like to thank Alex Whitworth, Dr. JE Treisman and the Bloomington Drosophila Stock Centre for the fly stocks, and Jalpa Parmar for the fly food preparation. We thank Inês P Castro, Nicoleta Moisoi and Roberta Tufi for reagents and advice. We also thank Dr. Aaron Voigt for sharing unpublished data. This work is funded by the Medical Research Council, UK. 
23. Brand $\mathrm{AH}$, Perrimon N. Targeted gene expression as a means of altering cell fates and generating dominant phenotypes. Development 1993; 118: 401-415.

1. de Castro IP, Martins LM, Tufi R. Mitochondrial quality control and neurological disease: an emerging connection. Expert Rev Mol Med 2010; 12: e12.

2. Baker MJ, Tatsuta T, Langer T. Quality control of mitochondrial proteostasis. Cold Spring Harb Perspect Biol 2011; 3: a007559.

3. Tatsuta T, Langer T. Quality control of mitochondria: protection against neurodegeneration and ageing. EMBO J 2008; 27: 306-314.

4. Haynes CM, Ron D. The mitochondrial UPR - protecting organelle protein homeostasis. $J$ Cell Sci 2010; 123: 3849-3855.

5. Twig G, Elorza A, Molina AJ, Mohamed H, Wikstrom JD, Walzer G et al. Fission and selective fusion govern mitochondrial segregation and elimination by autophagy. Embo $\mathrm{J}$ 2008; 27: 433-446.

6. Twig G, Hyde B, Shirihai OS. Mitochondrial fusion, fission and autophagy as a quality control axis: the bioenergetic view. Biochim Biophys Acta 2008; 1777: 1092-1097.

7. Wang K, Klionsky DJ. Mitochondria removal by autophagy. Autophagy 2011; 7: 297-300.

8. Youle RJ, Narendra DP. Mechanisms of mitophagy. Nat Rev Mol Cell Biol 2011; 12: 9-14.

9. Plun-Favreau H, Klupsch K, Moisoi N, Gandhi S, Kjaer S, Frith D et al. The mitochondrial protease HtrA2 is regulated by Parkinson's disease-associated kinase PINK1. Nat Cell Biol 2007; 9: 1243-1252.

10. Pridgeon JW, Olzmann JA, Chin LS, Li L. PINK1 protects against oxidative stress by phosphorylating mitochondrial chaperone TRAP1. PLoS Biol 2007; 5: e172.

11. Hua $G$, Zhang $Q$, Fan Z. Heat shock protein 75 (TRAP1) antagonizes reactive oxygen species generation and protects cells from granzyme M-mediated apoptosis. J Biol Chem 2007; 282: 20553-20560.

12. Masuda Y, Shima G, Aiuchi T, Horie M, Hori K, Nakajo S et al. Involvement of tumor necrosis factor receptor-associated protein 1 (TRAP1) in apoptosis induced by betahydroxyisovalerylshikonin. J Biol Chem 2004; 279: 42503-42515.

13. Montesano Gesualdi N, Chirico G, Pirozzi G, Costantino E, Landriscina M, Esposito F. Tumor necrosis factor-associated protein 1 (TRAP-1) protects cells from oxidative stress and apoptosis. Stress 2007; 10: 342-350.

14. Xiang $F$, Huang $Y S$, Shi $X H$, Zhang $Q$. Mitochondrial chaperone tumour necrosis factor receptor-associated protein 1 protects cardiomyocytes from hypoxic injury by regulating mitochondrial permeability transition pore opening. FEBS J 2010; 277: 1929-1938.

15. Costantino E, Maddalena F, Calise S, Piscazzi A, Tirino V, Fersini A et al. TRAP1, a nove mitochondrial chaperone responsible for multi-drug resistance and protection from apoptotis in human colorectal carcinoma cells. Cancer Lett 2009; 279: 39-46.

16. Landriscina M, Laudiero G, Maddalena F, Amoroso MR, Piscazzi A, Cozzolino F et al. Mitochondrial chaperone Trap1 and the calcium binding protein Sorcin interact and protect cells against apoptosis induced by antiblastic agents. Cancer Res 2010; 70: 6577-6586.

17. Lu B, Vogel H. Drosophila models of neurodegenerative diseases. Annu Rev Pathol 2009; 4: 315-342.

18. Clark IE, Dodson MW, Jiang C, Cao JH, Huh JR, Seol JH et al. Drosophila pink1 is required for mitochondrial function and interacts genetically with parkin. Nature 2006; 441: 1162-1166.

19. Park J, Lee SB, Lee S, Kim Y, Song S, Kim S et al. Mitochondrial dysfunction in Drosophila PINK1 mutants is complemented by parkin. Nature 2006; 441: 1157-1161.

20. Butler EK, Voigt A, Lutz AK, Toegel JP, Gerhardt E, Karsten $\mathrm{P}$ et al. The mitochondria chaperone protein TRAP1 mitigates alpha-Synuclein toxicity. Plos Genet2012; 8: e1002488.

21. Berry C, La Vecchia C, Nicotera P. Paraquat and Parkinson's disease. Cell Death Differ 2010; 17: 1115-1125.

22. Wills J, Jones J, Haggerty T, Duka V, Joyce JN, Sidhu A. Elevated tauopathy and alphasynuclein pathology in postmortem Parkinson's disease brains with and without dementia. Exp Neurol 2010; 225: 210-218.
24. Pimenta de Castro I, Costa AC, Lam D, Tufi R, Fedele V, Moisoi N et al. Genetic analysis of mitochondrial protein misfolding in Drosophila melanogaster. Cell Death Differ 2012; 19 1308-1316.

25. Verstreken P, Ly CV, Venken KJ, Koh TW, Zhou Y, Bellen HJ Synaptic mitochondria are critical for mobilization of reserve pool vesicles at Drosophila neuromuscular junctions. Neuron 2005; 47: 365-378.

26. Narendra D, Tanaka A, Suen DF, Youle RJ. Parkin is recruited selectively to impaired mitochondria and promotes their autophagy. J Cell Biol 2008; 183: 795-803.

27. Narendra DP, Jin SM, Tanaka A, Suen DF, Gautier CA, Shen J et al. PINK1 is selectively stabilized on impaired mitochondria to activate Parkin. PLOS Biol 2010; 8: e1000298.

28. Ziviani E, Tao RN, Whitworth AJ. Drosophila parkin requires PINK1 for mitochondrial translocation and ubiquitinates mitofusin. Proc Natl Acad Sci USA 2010; 107:

29. de Castro IP, Martins LM, Loh SH. Mitochondrial quality control and Parkinson's disease: a pathway unfolds. Mol Neurobiol 2011; 43: 80-86.

30. Riparbelli MG, Callaini G. The Drosophila parkin homologue is required for normal mitochondrial dynamics during spermiogenesis. Dev Biol 2007; 303: 108-120.

31. Deng H, Dodson MW, Huang H, Guo M. The Parkinson's disease genes pink1 and parkin promote mitochondrial fission and/or inhibit fusion in Drosophila. Proc Natl Acad Sci USA 2008; 105: 14503-14508.

32. Fuller MT. Spermatogenesis. In: Arias MBaAM (ed) The Development of Drosophila melanogaster pp 71-147, 1993

33. Tain LS, Chowdhury RB, Tao RN, Plun-Favreau H, Moisoi N, Martins LM et al. Drosophila $\mathrm{HtrA2}$ is dispensable for apoptosis but acts downstream of PINK1 independently from Parkin. Cell Death Differ 2009; 16: 1118-1125.

34. Moisoi N, Klupsch K, Fedele V, East P, Sharma S, Renton A et al. Mitochondrial dysfunction triggered by loss of $\mathrm{HtrA} 2$ results in the activation of a brain-specific transcriptional stress response. Cell Death Differ 2009; 16: 449-464.

35. Powers SK, Wiggs MP, Duarte JA, Zergeroglu AM, Demirel HA. Mitochondria signaling contributes to disuse muscle atrophy. Am J Physiol Endocrinol Metab 2012 303: E31-E39.

36. Lysakowski A, Figueras H, Price SD, Peng YY. Dense-cored vesicles, smooth endoplasmic reticulum, and mitochondria are closely associated with non-specialized parts of plasma membrane of nerve terminals: implications for exocytosis and calcium buffering by intraterminal organelles. J Comp Neurol 1999; 403: 378-390.

37. Greene JC, Whitworth AJ, Kuo I, Andrews LA, Feany MB, Pallanck LJ. Mitochondria pathology and apoptotic muscle degeneration in Drosophila parkin mutants. Proc Natl Acad Sci USA 2003; 100: 4078-4083.

38. Livak KJ, Schmittgen TD. Analysis of relative gene expression data using real-time quantitative PCR and the 2(-Delta Delta C(T)) Method. Methods 2001; 25: 402-408.

Cell Death and Disease is an open-access journal published by Nature Publishing Group. This work is licensed under the Creative Commons Attribution-NonCommercialShare Alike 3.0 Unported License. To view a copy of this license, visit http://creativecommons.org/licenses/by-nc-sa/3.0/ 5018-5023.

Supplementary Information accompanies this paper on Cell Death and Disease website (http://www.nature.com/cddis) 